\title{
The importance of evidence-based supportive care practice guidelines in childhood cancer-a plea for their development and implementation
}

\author{
E. A. H. Loeffen ${ }^{1}$ - L. C. M. Kremer ${ }^{2}$ R. L. Mulder ${ }^{2}$ - A. Font-Gonzalez ${ }^{2}$. \\ L. L. Dupuis ${ }^{3,4}$ - L. Sung ${ }^{5}$ • P. D. Robinson ${ }^{6}$ - M. D. van de Wetering ${ }^{2}$ - W. J. E. Tissing ${ }^{1}$
}

Received: 13 July 2016 / Accepted: 14 November 2016/Published online: 8 December 2016

(C) The Author(s) 2016. This article is published with open access at Springerlink.com

\begin{abstract}
As cure rates in pediatric oncology have improved substantially over the last decades, supportive care has become increasingly important to reduce morbidity and mortality and improve quality of life in children with cancer. Currently, large variations exist in pediatric oncology supportive care practice, which might negatively influence care. This plea underlines the importance of development and implementation of trustworthy supportive care clinical practice guidelines, which we believe is the essential next step towards better supportive care practice, and thus a higher quality of care. To facilitate international development and endorsement, the International Pediatric Oncology Guidelines in Supportive Care Network has been established.
\end{abstract}

Keywords Supportive care $\cdot$ Childhood cancer $\cdot$ Clinical practice guidelines $\cdot$ Evidence-based medicine

W. J. E. Tissing

w.j.e.tissing@umcg.nl

1 Department of Pediatric Oncology/Hematology, Beatrix Children's Hospital, University Medical Center Groningen, University of Groningen, PO Box 30.001, 9700, RB Groningen, the Netherlands

2 Department of Pediatric Oncology, Emma Children's Hospital, Academic Medical Center, Amsterdam, the Netherlands

3 Department of Pharmacy and Research Institute, The Hospital for Sick Children, Toronto, Canada

4 Leslie Dan Faculty of Pharmacy, University of Toronto, Toronto, Canada

5 Division of Haematology/Oncology, The Hospital for Sick Children, Toronto, Canada

6 Pediatric Oncology Group of Ontario, Toronto, Canada

\section{Main Text}

In the last century, medicine has changed drastically. In pediatric oncology, major changes in cure rates in developed countries have become visible since the introduction of chemotherapy in the late 1940s [1]. In the early 1960s, the five-year survival rate was $20 \%$, this increased to $40 \%$ in the 1970 s and is currently $80 \%$ in developed countries [2-4]. Although current survival rates are excellent, the ultimate goal is for all children to be cured of cancer. For instance, the mission statements of the St. Jude Children's Research Hospital (USA) and the recently founded Dutch national Princess Maxima Center for Pediatric Oncology both include the achievement of a greater than $90 \%$ childhood cancer overall survival rate in the next decade as their goal $[2,5]$.

The main reason for the improvement in survival is the introduction of intensive treatment protocols, encompassing chemotherapy, radiotherapy, and/or surgery [3]. Nonetheless, there is a cost associated with improved cure rates. This is among other things reflected in mortality due to treatmentrelated toxicity. Although progression or relapse of disease remains the number one cause of death, treatment-related toxicities also contribute significantly to mortality rates. For instance, a study in children with acute lymphoblastic leukemia found that one in four deaths in their cohort was due to treatment-related toxicity [6]. Furthermore, with cure rates improving, fewer children will die from the cancer itself and a higher proportion of deaths will be treatment-related [7].

Treatment-related mortality is not the only important metric of antineoplastic therapy-related toxicity; treatment-related morbidity also contributes substantially to poor quality of life and increased costs. Treatment-related morbidity includes various physical and psychosocial side effects as a consequence of the intensive treatment regimens.

To minimize short- and long-term morbidities and mortality due to adverse effects of treatment, optimal supportive care is 
extremely important. Supportive care in cancer is defined by the Multinational Association of Supportive Care in Cancer as "the prevention and management of the adverse effects of cancer and its treatment across the cancer continuum," and is thus all care provided to children with cancer, apart from the primary anti-cancer treatment (i.e. chemotherapy, radiotherapy, surgery, and novel treatment techniques such as targeted drugs) and follow-up care [8, 9]. Supportive care is very broad, comprising topics such as febrile neutropenia, nausea and vomiting, alopecia, nutrition, and psychosocial care. Important aims of present day pediatric oncology are to reduce short-term and long-term toxicities and to improve quality of life for both children who can and cannot be cured. Well-known examples are the introduction of central venous access devices and the introduction of anti-emetic prophylaxis. A challenge in supportive care is also handling adverse effects from supportive care itself, i.e., care that treats one problem but generates another one (usually less severe). For example, the introduction of broad-spectrum antibiotic prophylaxis on the one hand lowers the frequency of bacterial infections, but on the other hand may increase resistance rates and result in invasive fungal infections.

\section{Clinical practice guidelines}

To ensure that childhood cancer patients receive optimal care, the creation of high-quality evidence and the development of clinical practice guidelines are essential. In the last decade, there has been an increased interest in evidence-based guidelines. A clinical practice guideline (CPG) is defined as a "statement that includes recommendations intended to optimize patient care that are informed by a systematic review of evidence and an assessment of the benefits and harms of alternative care options" [10]. CPGs thus help the practitioner to provide the clinical care based upon the best available evidence.

However, despite all efforts, CPGs can differ greatly in terms of quality and applicability in practice. This is due to several reasons, for example, variation in methodology of development with different quality standards and hugely protracted development cycles which results in guidelines that are already outdated at moment of publication [11]. This led the Institute of Medicine to write the 2011 report entitled "Clinical Practice Guidelines We Can Trust," which made a substantiated plea for trustworthy CPGs [10]. In this report, eight standards (and 20 sub standards) are described for the development of trustworthy $\mathrm{CPG}$, which comprise transparency, management of conflicts of interest, composition of a development group, conducting a systematic review, establishing the evidence and rating the strength of recommendations, articulation of recommendations, external review, and updating. A set of similar standards are also described in a 2012 report by the Guidelines International Network (G-I$\mathrm{N})$, an international network of guideline developers, which underlines their importance [12]. Adhering to these standards in developing, and checking for these standards when endorsing, should lead to a base of trustworthy CPGs.

The Grading of Recommendations Assessment, Development, and Evaluation (GRADE) approach is an established approach in synthesizing evidence and developing recommendations [13]. The GRADE approach distinguishes two types of recommendations: strong and weak. For the clinician, this means that with a strong recommendation, most patients should receive this recommended course of action [14]. Thus, when CPGs are able to make strong recommendations, these statements provide guidance for physicians who are uncertain about the best approach to a clinical situation. Also, these CPGs may provide justification for funding or support in settings in which these are barriers to implementation. A weak recommendation implies that the clinician should realize that the appropriate choice differs per patient, and it is his or her role to inform the patient as to make a management decision that complements their personal values and preferences [14].

\section{Effect of clinical practice guidelines}

In many fields of medicine, care that is consistent with evidence-based guidelines has led to improved patient outcomes and more efficient care delivery [15-19]. Concerning supportive care in oncology, there is also evidence that promotes guideline-consistent care, for instance a recent study showed that adherence to an evidence-based guideline in relation to initiating antibiotic therapy in adult low-risk febrile neutropenia was associated with decreased mortality [20]. Another example is the reduction of chemotherapy-induced nausea and vomiting after highly or moderately emetogenic chemotherapy, which has been found repeatedly [21, 22].

CPGs have several advantages, of which their potential to improve patient health outcomes is undoubtedly the greatest [23]. Also, the current vast amount of literature makes it impossible for clinicians to absorb and synthesize all available evidence, a CPG facilitates herein and makes recommendations that are consistent with this evidence. Other advantages of CPGs include improvement of structure and consistency of care, improvement of patient knowledge and awareness (e.g. through patient versions/leaflets), enhancement of public policy with regard to e.g. preventive interventions, and exposure of gaps in contemporary scientific knowledge [18].

\section{Clinical practice guidelines in pediatric oncology}

A 2015 systematic search of MEDLINE and Embase for recent evidence-based supportive care practice guidelines relevant to childhood cancer identified 17 guidelines, published in English in 2012 or more recently, that met or provisionally met the Institute of Medicine's 2011 definition of being based on a systematic review (Robinson PD, Seelisch J, Kelly M, Madden J, Kelly K, Thackray J, et al., 2015, Identification of 
evidencebased guidelines for pediatric cancer supportive care: a systematic review, unpublished results, in progress). Of these, only four were specifically aimed at children with cancer, addressing febrile neutropenia, chemotherapy-induced nausea and vomiting, and antifungal prophylaxis [24-27]. However, for the majority of supportive care topics in pediatric oncology, there are no high-quality, recent evidence-based guidelines available to assist clinicians.

There might be several reasons underlying the scarcity of childhood cancer supportive care guidelines. For one, in the past decades, the majority of the pediatric oncology research has focused on improving cure rates, which led to the remarkable increase to a current five-year survival rate of $80 \%$ as mentioned earlier [4]. Now that cure is so often achieved, we believe that we should also turn to the field of supportive care and that supportive care parameters and quality of life should be considered more often as primary clinical and research endpoints. Another reason underlying suboptimal supportive care is that standardization for supportive care practice is lacking [28]. For instance, a recent study found $75 \%$ discordant supportive care practice among all Dutch pediatric cancer centers, also in topics for which a CPG was available (e.g. nausea and vomiting) [29]. In contrast, for primary oncological treatment, all Dutch pediatric cancer hospitals have endorsed nationwide protocols, which are set up as large (inter)national clinical trials. This further increases the specific need for supportive care CPGs.

Developing CPGs does not automatically guarantee an improvement in the quality of care and health outcomes. Studies have shown that 30 to $50 \%$ of patients do not receive the recommended best practice [30,31]. In addition to a highquality guideline development methodology, the dissemination and implementation strategies are of major importance for the successful uptake of CPGs [30]. Besides the development of implementation strategies, it is important to evaluate whether the CPG is actually used in practice and if it contributes to an improvement in care. Therefore, it is important to develop quality indicators to measure the adherence to the CPGs [32]. In addition, development of CPGs provides an overview of the current available evidence, while exposing the current gaps in knowledge. This can be of great use when defining a research agenda for the upcoming years.

\section{iPOG network}

To take maximum advantage of international effort and expertise in developing and implementing supportive care CPGs, the International Pediatric Oncology Guidelines in Supportive Care Network (iPOG Network) has been established [33]. This voluntary international collaboration invites all who are developing or endorsing supportive care clinical practice guidelines in pediatric oncology to join. Current members comprise representatives from Children's Cancer \& Blood Disorders (C17, Canada), Children's Cancer and Leukemia
Group (CCLG, United Kingdom), Children's Oncology Group (COG, United States of America), Dutch Childhood Oncology Group (DCOG, the Netherlands), Pediatric Oncology Group of Ontario (POGO, Canada), and The American Society of Pediatric Hematology/Oncology (ASPHO, United States of America).

The goal of iPOG is fourfold: (1) sharing best practices in CPG development, (2) avoiding inadvertent duplication of effort by sharing guideline work plans and endorsement processes, (3) communicating evidence gaps to the research community, and (4) providing a forum for discussions regarding guideline dissemination and implementation.

The website of the POOG network is part of the website of The Hospital for Sick Children (SickKids, Toronto, Canada) and can be found at: http://www.sickkids.ca/Research/iPOG/. Both the CPGs that are available through the iPOG network and those currently in progress are shown in Table 1.

Table 1 Overview of CPGs of the iPOG network that are currently available and those in progress

Available CPGs through the iPOG network

Antiemesis

Guideline for classification of the acute emetogenic potential of antineoplastic medication in pediatric cancer patients [34], 2011

Prevention of acute nausea and vomiting due to antineoplastic medication in pediatric cancer patients [25], 2013

Prevention and treatment of anticipatory nausea and vomiting due to chemotherapy in pediatric cancer patients [24], 2014

Guideline for the treatment of breakthrough and the prevention of refractory chemotherapy-induced nausea and vomiting in children with cancer [35], 2016

Infectious diseases

Guideline for the management of fever and neutropenia in children with cancer and/or undergoing hematopoietic stem cell transplantation [26], 2012

Guideline for primary antifungal prophylaxis for pediatric patients with cancer or hematopoietic stem cell transplant recipients [27], 2014

Guideline for the prevention of oral and oropharyngeal mucositis in children receiving treatment for cancer or undergoing hematopoietic stem cell transplantation [36], 2015

CPGs currently in progress in the iPOG network

Dexrazoxane use in children (expected: 2017)

Fertility preservation for female pediatric cancer patients (PanCareLIFE, expected: 2017)

Fertility preservation for male pediatric cancer patients (PanCareLIFE, expected: 2017)

Pain management in pediatric cancer patients (expected: 2017)

Pneumocystits jirovecii prophyalxis in pediatric cancer patients (expected: 2017)

Prevention of acute chemotherapy-induced nausea and vomiting; focused update on aprepitant and palonosetron (expected: 2017)

School re-integration for children receiving cancer treatment (expected: 2017) 


\section{Conclusion}

With increasing survival, supportive care has become more and more important in improving both short- and long-term morbidities and mortality in children with cancer. We believe that the development and use of supportive care CPGs are the imperative next steps in bridging the gap between current evidence and daily practice. Although significant work has been done already, there are still many topics in supportive care in pediatric oncology that can and should be covered [37]. In this way, we can reduce morbidity and mortality in children with cancer. International collaboration is essential to avoid duplication in work and to be efficient. The groups working together in the iPOG network will develop and implement trustworthy supportive care CPGs on topics which are important to clinicians, families, and children with cancer. We invite all who are willing to contribute to this initiative to collaborate.

\section{Compliance with ethical standards}

Conflict of interest The authors declare that they have no conflict of interest.

Funding The project "Towards evidence-based guidelines for supportive care in childhood oncology" is supported by the Alpe d'HuZes foundation/Dutch Cancer Society (RUG 2013-6345).

Open Access This article is distributed under the terms of the Creative Commons Attribution-NonCommercial 4.0 International License (http:// creativecommons.org/licenses/by-nc/4.0/), which permits any noncommercial use, distribution, and reproduction in any medium, provided you give appropriate credit to the original author(s) and the source, provide a link to the Creative Commons license, and indicate if changes were made.

\section{References}

1. DeVita VT, Chu E (2008) A history of cancer chemotherapy. Cancer Res 68:8643-8653

2. About Us - St. Jude Children's Research Hospital [Internet]. Memphis, TN, United States of America: St. Jude Children's Research Hospital. [cited 2015 December 22nd]. Available from: https://www.stjude.org/about-st-jude.html

3. Kaatsch P (2010) Epidemiology of childhood cancer. Cancer Treat Rev 36:277-285

4. Gatta G, Botta L, Rossi S, Aareleid T, Bielska-Lasota M, Clavel J et al (2014) Childhood cancer survival in Europe 1999-2007: results of EUROCARE-5-a population-based study. Lancet Oncol 15: 35-47

5. Doelstellingen - Prinses Máxima Centrum [Internet]. Utrecht, the Netherlands: Prinses Máxima Centrum. [cited 2015 December 22nd]. Available from: http://www.prinsesmaximacentrum. $\mathrm{nl} /$ over-ons/hoe-en-waarom/doelstellingen/

6. Lund B, Åsberg A, Heyman M, Kanerva J, Harila-Saari A, Hasle H et al (2011) Risk factors for treatment related mortality in childhood acute lymphoblastic leukaemia. Pediatr Blood Cancer 56:551-559
7. Ethier MC, Blanco E, Lehrnbecher T, Sung L (2011) Lack of clarity in the definition of treatment-related mortality: pediatric acute leukemia and adult acute promyelocytic leukemia as examples. Blood 118:5080-5083

8. Sung L, Zaoutis T, NJ U, Johnston D, Dupuis L, Ladas E, Children's Oncology Group's (2013) Blueprint for research: cancer control and supportive care. Pediatr Blood Cancer 60:1027-1030

9. MASCC - Multinational Association of Supportive Care in Cancer [Internet]. Hillerød, Denmark: Multinational Association of Supportive Care in Cancer. [cited 2016 January 27th]. Available from: http://www.mascc.org/

10. Graham R, Mancher M, Wolman DM (2011) Clinical practice guidelines We can trust. The National Academies Press, Washington D.C

11. Classen DC, Mermel LA (2015) Specialty society clinical practice guidelines time for evolution or revolution? JAMA 314:871-872

12. Qaseem A, Forland F, Macbeth F, Ollenschläger G, Phillips S, van der Wees P (2012) Guidelines international network: toward international standards for clinical practice guidelines. Ann Intern Med 156:525-531

13. Guyatt GH, Oxman AD, Vist GE, Kunz R, Falck- Y, Alonso-coello $P$ et al (2008) GRADE: an emerging consensus on rating quality of evidence and strength of recommendations. BMJ 336:924-926

14. Guyatt GH, Oxman AD, Kunz R, Falck-Ytter Y, Vist GE, Liberati A et al (2008) GRADE: going from evidence to recommendations. BMJ 336:1049-1051

15. Ansari S, Rashidian A (2012) Guidelines for guidelines: are they up to the task? A comparative assessment of clinical practice guideline development handbooks. PLoS One 7:e49864

16. Grimshaw J, Russell I (1993) Effect of clinical guidelines on medical practice: a systematic review of rigorous evaluations. Lancet 342:1317-1322

17. Grimshaw JM, Thomas RE, MacLennan G, Fraser C, Ramsay CR, Vale L et al (2004) Effectiveness and efficiency of guideline dissemination and implementation strategies. Health Technol Assess $8: 1-72$

18. Lugtenberg M, Burgers JS, Westert GP (2009) Effects of evidencebased clinical practice guidelines on quality of care: a systematic review. Qual Saf Health Care 18:385-392

19. Inwald EC, Ortmann O, Zeman F, Koller M, Hofstädter F, Gerstenhauer $M$ et al (2014) Guideline concordant therapy prolongs survival in HER2-positive breast cancer patients: results from a large population-based cohort of a cancer registry. Biomed Res Int 2014:137304

20. Wright JD, Neugut AI, Ananth CV, Lewin SN, Wilde ET, Lu Y-S et al (2013) Deviations from guideline-based therapy for febrile neutropenia in cancer patients and their effect on outcomes. JAMA Intern Med 173:559-568

21. Aapro M, Molassiotis A, Dicato M, Pelaez I, Rodriguez-Lescure A, Pastorelli D et al (2012) The effect of guideline-consistent antiemetic therapy on chemotherapy-induced nausea and vomiting (CINV): the Pan European Emesis Registry (PEER). Ann Oncol 23:19861992

22. Gilmore BJW, Peacock NW, Gu A, Szabo S, Rammage M, Sharpe J et al (2013) Antiemetic guideline consistency and incidence of chemotherapy-induced nausea and vomiting in US community oncology practice: INSPIRE study. J Oncol Pract 10:68-74

23. Woolf SH, Grol R, Hutchinson A, Eccles M, Grimshaw J (1999) Clinical guidelines: potential benefits, limitations, and harms of clinical guidelines. BMJ 318:527-530

24. Dupuis LL, Robinson PD, Boodhan S, Holdsworth M, Portwine C, Gibson P et al (2014) Guideline for the prevention and treatment of anticipatory nausea and vomiting due to chemotherapy in pediatric cancer patients. Pediatr Blood Cancer 61:1506-1512

25. Dupuis LL, Boodhan S, Holdsworth M, Robinson PD, Hain R, Portwine C et al (2013) Guideline for the prevention of acute nausea 
and vomiting due to antineoplastic medication in pediatric cancer patients. Pediatr Blood Cancer 60:1073-1082

26. Lehrnbecher T, Phillips R, Alexander S, Alvaro F, Carlesse F, Fisher B et al (2012) Guideline for the management of fever and neutropenia in children with cancer and/or undergoing hematopoietic stem-cell transplantation. J Clin Oncol 30:4427-4438

27. Science M, Robinson PD, MacDonald T, Rod Rassekh S, Dupuis LL, Sung L (2014) Guideline for primary antifungal prophylaxis for pediatric patients with cancer or hematopoietic stem cell transplant recipients. Pediatr Blood Cancer 64:393-400

28. Mueller BU (2014) Quality and safety in pediatric hematology/oncology. Pediatr Blood Cancer 61:966-969

29. Loeffen EAH, Mulder RL, van de Wetering MD, Font-Gonzalez A, Abbink FCH, Ball LM et al (2015) Current variations in childhood cancer supportive Care in the Netherlands. Cancer 122):642-650

30. Prior M, Guerin M, Grimmer-Somers K (2008) The effectiveness of clinical guideline implementation strategies - a synthesis of systematic review findings. J Eval Clin Pract 14:888-897

31. Grol R, Grimshaw J (2003) From best evidence to best practice: effective implementation of change in patients' care. Lancet 362:1225-1230

32. Campbell SM (2002) Research methods used in developing and applying quality indicators in primary care. Qual Saf Heal Care $11: 358-364$
33. International Pediatric Oncology Guidelines in Supportive Care Network (iPOG Network) [Internet]. Toronto, Canada: The Hospital for Sick Children (SickKids). [cited 2015 December 22nd] Available from: http://www.sickkids.ca/Research/iPOG/

34. Dupuis LL, Boodhan S, Sung L, Portwine C, Hain R, McCarthy P et al (2011) Guideline for the classification of the acute emetogenic potential of antineoplastic medication in pediatric cancer patients. Pediatr Blood Cancer 57:191-198

35. Flank J, Robinson PD, Holdsworth M, Phillips R, Portwine C, Gibson P et al (2016) Guideline for the treatment of breakthrough and the prevention of refractory chemotherapy-induced nausea and vomiting in children with cancer. Pediatr Blood Cancer 63:1144 1151

36. Sung L, Robinson P, Treister N, Baggott T, Gibson P, Tissing W et al (2015) Guideline for the prevention of oral and oropharyngeal mucositis in children receiving treatment for cancer or undergoing haematopoietic stem cell transplantation. BMJ Support Palliat Care. doi:10.1136/bmjspcare-2014-000804

37. Loeffen EAH, Mulder RL, Kremer LCM, Michiels EMC, Abbink FCH, Ball LM et al (2015) Development of clinical practice guidelines for supportive care in childhood cancer-prioritization of topics using a Delphi approach. Support Care Cancer 23(7):19871995 